\title{
The number of monounary algebras
}

\author{
Gábor Horváth, Kamilla Kátai-Urbán, Péter Pál Pach, \\ Gabriella Pluhár, András Pongrácz, and Csaba Szabó
}

\begin{abstract}
In this note we give an asymptotic estimate for the number of monounary algebras of given size.
\end{abstract}

\section{Introduction}

A monounary algebra is an algebra with a single unary operation. The theory of monounary algebras is well-developed, for a recent monograph see [2]. Let $\mathcal{A}=(A, f)$ be a monounary algebra. The function $f$ defines a directed graph on $A$. Let $G_{A}=(A, E)$, the vertex set is $A$ and the edges are $E=$ $\{(a, f(a)) \mid a \in A\}$. In $G_{A}$ every vertex has outdegree 1 , and every graph $G$ with outdegree 1 defines a monounary algebra on its vertex set, where $f(a)$ is the single vertex such that $(a, f(a))$ is an edge in $G$. Hence, there is a bijection between monounary algebras and directed graphs, where each vertex has outdegree 1. At first, we investigate the number of finite connected nonisomorphic monounary algebras.

Theorem 1.1. Let $C_{n}$ denote the number of connected non-isomorphic monounary algebras of size $n$. Then there is an $\alpha>1$ such that $\log _{\alpha} C_{n} \sim n$.

Proof. A directed graph corresponding to a connected monounary algebra is a directed cycle with a (possibly one-element) rooted tree at each vertex of the cycle. If there is a loop in the graph of the algebra, we say that the length of the cycle is 1. Each edge of each rooted tree is directed towards the cycle, and the cycle consists of the roots of the trees. Hence, a connected graph of a monounary algebra of size $n$ is built up from a cycle of length $k$, where $1 \leq k \leq n$ and to each vertex of the cycle we glue a rooted tree such that the sum of the sizes of the rooted trees is $n$. Every connected monounary algebra of size $n$ can be obtained in this way. Note that these graphs are not necessarily non-isomorphic. Naturally, to each rooted tree there corresponds a unique monounary algebra with a one-element cycle. Let $T_{n}$ denote the number of rooted trees on $n$ vertices. By the above arguments we have

2010 Mathematics Subject Classification: 08A60, 05A16.

Key words and phrases: monounary algebra, generating function.

The research of the authors was supported by the Hungarian National Foundation for Scientific Research Grant K67870 and K83219. 


$$
T_{n} \leq C_{n} \leq \sum_{k=1}^{n} \sum_{i_{1}+\cdots+i_{k}=n} T_{i_{1}} T_{i_{2}} \cdots T_{i_{k}}
$$

Let $T_{0}=C_{0}=1$. Let $\psi(x)=\sum_{n=1}^{\infty} T_{n} x^{n-1}$ and $\Gamma(x)=\sum_{n=0}^{\infty} C_{n} x^{n}$. Now $\Gamma$ is the generating function of the number of connected monounary algebras, and $x \psi(x)+1$ is the generating function of the number of rooted trees. By formula (1) the following holds:

$$
x \psi(x)+1 \leq \Gamma(x) \leq 1+\sum_{n=1}^{\infty}\left(\sum_{k=1}^{n} \sum_{i_{1}+\cdots i_{k}=n} T_{i_{1}} T_{i_{2}} \ldots T_{i_{k}}\right) x^{n}=\sum_{n=0}^{\infty}(x \psi(x))^{n}
$$

In [3] the function $\psi(x)$ is well analyzed. It is proved that there is a constant $c_{T}$ such that $T_{n} \sim c_{T} \alpha^{n} n^{-\frac{3}{2}}$, where $\alpha \sim 2.955765$. Moreover, the power series $\psi(x)$ has radius of convergence $\frac{1}{\alpha}$ for the same $\alpha$, and $\psi\left(\frac{1}{\alpha}\right)=\alpha$, hence $\frac{1}{\alpha} \psi\left(\frac{1}{\alpha}\right)=1$ holds. As $\sum_{n=0}^{\infty}(x \psi(x))^{n}=\frac{1}{1-x \psi(x)}$ is strictly monotonically increasing in $\mathbb{R}^{+}$, the radius of convergence of this power series is the unique positive solution of the equation $x \psi(x)=1$, which is $\frac{1}{\alpha}$. Therefore for each power series in (2) the radius of convergence is $\frac{1}{\alpha}$. This holds for $\Gamma(x)$, as well. Thus for the coefficients of $\Gamma(x)$ we have $\limsup \sqrt[n]{C_{n}}=\alpha$. From $T_{n} \leq C_{n}$ we obtain $\liminf _{n \rightarrow \infty} \sqrt[n]{C_{n}} \geq \alpha$, thus $\lim _{n \rightarrow \infty} \sqrt[n]{C_{n}}=\alpha$, and $\log _{\alpha} C_{n} \sim n$ is gained.

Note that asymptotic tree counting techniques in which generating functions were applied, were investigated in more detail also in [1].

Theorem 1.2. Let $M_{n}$ denote the number of monounary algebras of size $n$. Then $\log _{\alpha} M_{n} \sim n$.

Proof. A monounary algebra is the disjoint union of connected monounary algebras. Let $\mathcal{A}$ be a monounary algebra of size $n$ consisting of $p_{i}$ connected algebras of size $i$. Then $\sum_{i=1}^{n} i p_{i}=n$. Note that $p_{i}$ can be 0 . The number of ways of picking $k$ indistinguishable objects of type $p$ is the coefficient of $x^{k}$ in the generating function $(1-x)^{-p}$. Hence, the number of ways picking $p_{i}$ indistinguishable connected monounary algebras of type $C_{i}$ is the coefficient of $x^{i p_{i}}$ in $\left(1-x^{i}\right)^{-C_{i}}$. Thus the generating function for the number of monounary algebras is

$$
1+\sum_{n=1}^{\infty} M_{n} x^{n}=\prod_{k=1}^{\infty} \frac{1}{\left(1-x^{k}\right)^{C_{k}}}
$$


For $x>0$ this series converges if and only if

$$
\log \left(\prod_{k=1}^{\infty} \frac{1}{\left(1-x^{k}\right)^{C_{k}}}\right)=\sum_{k=1}^{\infty}-C_{k} \log \left(1-x^{k}\right)
$$

is convergent. If $x \in\left(0, \frac{1}{\alpha}\right)$ then $x^{k} \in\left(0, \frac{1}{\alpha}\right)$ for all $k \geq 1$. As $\log x$ is concave we have $\log (1-t) \geq-c t$ for all $t \in\left(0, \frac{1}{\alpha}\right)$ with $c=-\alpha \log \left(1-\frac{1}{\alpha}\right)$. Therefore $\sum_{k=1}^{\infty}-C_{k} \log \left(1-x^{k}\right) \leq \sum_{k=1}^{\infty} C_{k} c x^{k}=c(\Gamma(x)-1)$. Hence this power series - and consequently $\sum_{n=0}^{\infty} M_{n} x^{n}-$ is convergent in $\left(0, \frac{1}{\alpha}\right)$. This yields $\limsup _{n \rightarrow \infty} \sqrt[n]{M_{n}} \leq \alpha$. The lower bound $\liminf _{n \rightarrow \infty} \sqrt[n]{M_{n}} \geq \alpha$ can be derived from $C_{n} \leq M_{n}$. Thus $\lim _{n \rightarrow \infty} \sqrt[n]{M_{n}}=\alpha$ and $\log _{\alpha} M_{n} \sim n$.

\section{REFERENCES}

[1] M. Drmota, Random trees. An interplay between combinatorics and probability, Springer, Wien, 2009 pp. 458

[2] D. Jakubíková-Studenovská, J. Pócs, Monounary Algebras, UPJS, Kosice, 2009 pp. 304

[3] R. Otter, The number of trees, Annals of Mathematics 49 (1948), no. 3, 583-599.

GÁBOR Horváth

1 Institute of Mathematics, University of Debrecen, Pf. 12, Debrecen, 4010, Hungary

Kamilla Kátai-URBÁN

2 Bolyai Institute, University of Szeged, Aradi vértanúk tere 1, H-6720 Szeged, Hungary e-mail: ghorvath@math.unideb.hu

Péter PÁl PACH

3,4,6 Eötvös Loránd University, Department of Algebra and Number Theory, 1117 Budapest, Pázmány Péter sétány 1/c, Hungary

e-mail: katai@math.u-szeged.hu

Gabriella Pluhár and András Pongrácz

5 Central European University, Budapest, Hungary

e-mail: ppp24@cs.elte.hu

e-mail: plugab@cs.elte.hu

e-mail: csaba@cs.elte.hu 